\title{
Enhancement of the sensitivity of a temperature sensor based on fiber Bragg gratings via weak value amplification
}

\author{
L. J. Salazar-Serrano, ${ }^{1,2, *}$ D. Barrera,${ }^{3}$ W. Amaya, ${ }^{1}$ S. Sales, ${ }^{3}$ V. Pruneri, ${ }^{1}$ \\ J. Capmany, ${ }^{3}$ and J. P. Torres ${ }^{1,4}$ \\ ${ }^{1}$ ICFO-Institut de Ciencies Fotoniques, Mediterranean Technology Park, 08860 Castelldefels (Barcelona), Spain \\ ${ }^{2}$ Quantum Optics Laboratory, Universidad de los Andes, AA 4976, Bogotá, Colombia \\ ${ }^{3}$ iTEAM Institute, Universidad Politecnica de Valencia, 46022 Valencia, Spain \\ ${ }^{4}$ Department of Signal Theory and Communications, Universitat Politecnica de Catalunya, 08034 Barcelona, Spain \\ *Corresponding author: luis-jose.salazar@icfo.es
}

Received 9 June 2015; revised 27 July 2015; accepted 31 July 2015; posted 3 August 2015 (Doc. ID 242435); published 19 August 2015

We present a proof-of-concept experiment aimed at increasing the sensitivity of Fiber-Bragg-gratings temperature sensors by making use of a weak-value-amplification scheme. The technique requires only linear optics elements for its implementation and appears as a promising method for increasing the sensitivity than state-of the-art sensors can currently provide. The device implemented here is able to generate a shift of the centroid of the spectrum of a pulse of $\sim 0.035 \mathrm{~nm} /{ }^{\circ} \mathrm{C}$, a nearly fourfold increase in sensitivity over the same fiber-Bragg-grating system interrogated using standard methods. (C) 2015 Optical Society of America

OCIS codes: (140.3490) Lasers, distributed-feedback; (120.6780) Temperature; (060.2370) Fiber optics sensors; (120.3180) Interferometry.

http://dx.doi.org/10.1364/OL.40.003962

Fiber Bragg Gratings (FBG) constitute nowadays a key ingredient of many devices used in communication and sensing applications [1]. They can easily be integrated in all-fiber systems, their dielectric nature make them nonconducting and immune to electromagnetic interference, and current technology allows to tailor the properties of FBGs to adapt to the specific requirements of each application.

When considering a FBG as a sensor, it can be understood as a bandpass filter whose central frequency depends on the value of a variable (temperature or strain). Thus the achievement of increasingly higher sensitivity in FBG-based systems implies the development of new techniques to enhance the shift of the central frequency of the filter for a given change of temperature or strain.

Here, we consider the use of a technique generally referred as weak-value amplification (WVA), a concept first introduced by Aharonov et al. [2]. It is a signal-enhancement (amplification) technique that is used in metrology applications to measure tiny changes of a variable that cannot be detected otherwise because of technical limitations, i.e., the insufficient sensitivity of the detection system. It makes use of the weak coupling that is introduced between two degrees of freedom of a system. Here, the weak coupling takes place between the shift of the centroid of the spectrum of a pulse and its polarization.

The concept of weak-value amplification can be readily understood in terms of constructive and destructive interference between probability amplitudes in a quantum mechanics context [3], or in terms of interference of classical waves $[4,5]$. Indeed, most of the experimental implementations of the concept, since its first demonstration in 1991 [6], belong to the last type. In this scenario, the usefulness of weak-value amplification for measuring extremely small quantities has been demonstrated under a great variety of experimental conditions [7-11].

The use of FBGs as temperature sensors has been considered $[12,13]$. Recently the WVA concept was applied to the demonstration of a laser-based thermostat, based on the measurement of the temperature-induced deviation of a laser when traversing a fluid with a high thermo-optic coefficient [14]. In this Letter, we show the usefulness of this technique when applied to temperature sensing based on the use of FBGs. We should notice that while we consider a temperature sensor, other characteristics as well, such as strain can also be considered as targets. In this respect, the method is general and can be applied to measuring all kinds of physical parameters, provided they could generate a measurable change of the response of FBGs when interrogated by appropriate light beams.

The system considered makes use of a broad-band light source, two FBGs at slightly different temperatures, and an optical spectrum analyzer (OSA), all of them interconnected by optical circulators and optical fibers. The FBG reflects only a small portion of the input spectrum centered at a certain value determined by the combination of the temperature and strain to which the device is subjected. For configurations where the FBG is isolated from any source of strain, the position of the 
centroid of the spectrum of the reflected light varies linearly with respect to the temperature with a sensitivity ranging from $0.008 \mathrm{~nm} /{ }^{\circ} \mathrm{C}$ to $0.014 \mathrm{~nm} /{ }^{\circ} \mathrm{C}$ determined by the material of the fiber. As a result, spectrum analyzers with high resolution are required to measure temperatures changes below 1 degree centigrade. Here we demonstrate a system that can reach a sensitivity of up to $\sim 0.035 \mathrm{~nm} /{ }^{\circ} \mathrm{C}$ when WVA is used, to be compared with a sensitivity of $\sim 0.009 \mathrm{~nm} /{ }^{\circ} \mathrm{C}$ that we measure without the use of WVA. This enhancement allows to measure smaller temperature difference given a specific sensitivity of the OSA.

Figure 1(a) depicts the experimental scheme implemented. A laser generates pulses with central frequency $\nu_{0}$ (central wavelength: $1549 \mathrm{~nm}$ ) that are linearly polarized at $+45^{\circ}$ by using a linear polarizer $\left(\mathrm{POL}_{1}\right)$. This constitutes the pre-selection stage. The laser is a femtosecond fiber laser (Calmar LaserMendocino) that generates $320 \mathrm{fs}$ pulses (bandwidth: $11 \mathrm{~nm}$ ) with a Gaussian-like spectrum, average power $3 \mathrm{~mW}$ and repetition rate $20 \mathrm{MHz}$.

The two orthogonal polarizations ( $H$ and $V$ ) are divided by a polarizing beam splitter $\left(\mathrm{PBS}_{1}\right)$ and follow different paths. The signal in each path is connected to $\mathrm{FBGs}\left(\mathrm{FBG}_{1}\right.$ and $\mathrm{FBG}_{2}$ ) by means of circulators $\left(\mathrm{CIRC}_{1}\right.$ and $\left.\mathrm{CIRC}_{2}\right)$. The signal traversing each arm of the interferometer is focused into a single-mode (SM) fiber that is connected to the first port the circulator. The second port is connected to the FBG that filters out the input signal with an efficiency of $14 \%$, and leaves

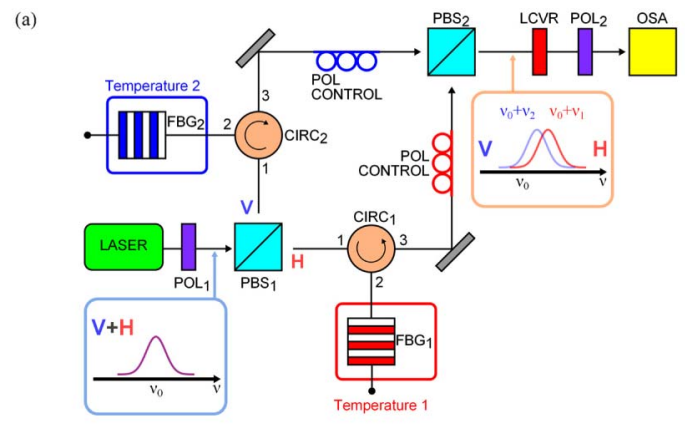

(b)
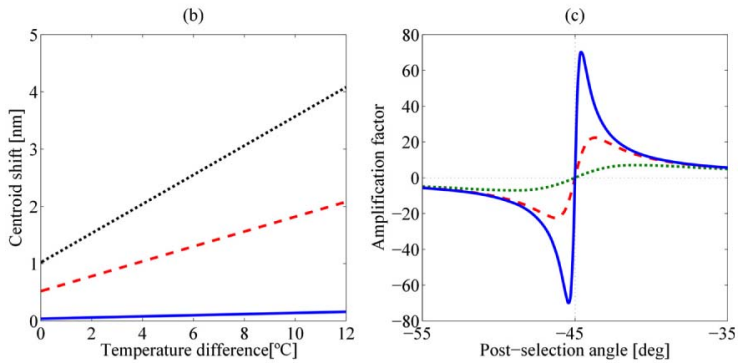

Fig. 1. (a) Experimental scheme: $\mathrm{FBG}_{1}$ and $\mathrm{FBG}_{2}$, fiber Bragg gratings; $\mathrm{POL}_{1}$ and $\mathrm{POL}_{2}$, polarizers; $\mathrm{PBS}_{1}$ and $\mathrm{PBS}_{2}$, polarizing beam splitters; LCVR, liquid-crystal variable retarder; OSA, optical spectrum analyzer; $\mathrm{CIRC}_{1}$ and $\mathrm{CIRC}_{2}$, optical circulators. (b) Theoretical shift of the centroid of the output spectrum as a function of the temperature difference $T_{1}-T_{2}$ for three different post-selection polarizations, and thus amplification factors. $\beta=0^{\circ}$ and $\mathcal{A}=1$ (blue continuous line), $\beta=-42.8^{\circ}$ and $\mathcal{A}=25$ (red dashed line), and $\beta=$ $-44.02^{\circ}$ and $\mathcal{A}=50$ (black dotted line). (c) Theoretical amplification factor as a function of the post-selection angle $\beta$ for different values of $\gamma_{\nu} \gamma_{\tau} \cos \delta$ : 0.9999 (continuous line), 0.999 (dashed-line), and 0.99 (dotted-line). the FBG with a Gaussian-like spectrum, $2.5 \mathrm{~nm}$ wide full width at half-maximum (FWHM), centered at $\approx 1551 \mathrm{~nm}$.

Each FBG is embedded into an oven that is set to a different temperature, $T_{1}$ and $T_{2}$. The FBG acts as a filter whose central frequency is determined by the temperatures of the corresponding oven. If the bandwidth of the input pulse is larger than the bandwidth of the FBG, the effect of a temperature difference is to generate two similar pulses with orthogonal polarizations and different central frequencies. The third port of each circulator is connected to a collimator lens that launches the output beams toward a second $\mathrm{PBS}\left(\mathrm{PBS}_{2}\right)$, that combines the two pulses into a single beam.

Before reaching $\left(\mathrm{PBS}_{2}\right)$, and due to polarization changes introduced by the circulators and FBGs, the state of polarization of each pulse is rectified after leaving the circulators using polarization controllers. In this way, we assure that before recombining again the two pulses in $\mathrm{PBS}_{2}$, the pulse that traversed $\mathrm{FBG}_{1}$ is horizontally polarized, and its central frequency is $\nu_{1}$, whereas the pulse that traversed $\mathrm{FBG}_{2}$ is vertically polarized and its spectrum is centered at $\nu_{2}$. In all cases, we are interested in detecting small temperature changes, so that the frequency shift $\nu_{1}-\nu_{2}$ is smaller than the FBG bandwidth $(B)$. To compensate the phase introduced due to birefringence in the circulators and single-mode (SM) fibers, a liquid crystal variable retarder (LCVR) (Thorlabs-LCC1113-C) is added after $\mathrm{PBS}_{2}$.

After $\mathrm{PBS}_{2}$, the electric field in the frequency domain reads

$$
\begin{aligned}
\mathbf{E}(\nu)= & \frac{E_{0}}{\sqrt{2}}\left\{\hat{x} \exp \left[-\frac{\left(\nu-\nu_{0}-\nu_{1}\right)^{2}}{2 B^{2}}\right]\right. \\
& \left.+\hat{y} \exp \left[-\frac{\left(\nu-\nu_{0}-\nu_{2}\right)^{2}}{2 B^{2}}+i(2 \pi \nu \tau+\delta)\right]\right\}
\end{aligned}
$$

where $\hat{x}$ and $\hat{y}$ designate horizontal and vertical polarization, respectively, $\tau$ takes into account the optical path difference present in the experimental setup, and $B^{2}=\ln 2 / \pi^{2} T^{2}$, with $T$ being the temporal duration (full width half-maximum) of the pulses reflected from the FBGs. $\delta=\phi-\Gamma$, where $\phi$ denotes a phase due to the birefringence induced from bends and twists in circulators and single-mode fibers and $\Gamma$ is a phase introduced with a liquid crystal variable retarder (LCVR) to compensate the unwanted phase $\phi$. Inspection of Eq. (1) shows clearly the coupling between the shift of the centroid of the spectrum of each pulse, $\nu_{1}$ and $\nu_{2}$, and its polarization, a key element of the WVA scheme.

The weak value amplification effect is introduced by projecting the recombined signal into a polarization state $\mathbf{e}_{\text {out }}=$ $\cos \beta \hat{x}+\sin \beta \hat{y}$ with the help of a second polarizer $\left(\mathrm{POL}_{2}\right)$ that is rotated using a motorized rotation stage. This is the post-selection stage. After the post-selection, the output beam is collimated with a SM fiber connected to an optical spectrum analyzer (OSA).

The power spectrum $(S)$ measured with the OSA, after postselection, reads

$$
\begin{aligned}
S(\nu)= & \frac{S_{0}}{2}\left\{\cos ^{2} \beta \exp \left[-\frac{\left(\nu-\nu_{0}-\nu_{1}\right)^{2}}{B^{2}}\right]\right. \\
& +\sin ^{2} \beta \exp \left[-\frac{\left(\nu-\nu_{0}-\nu_{2}\right)^{2}}{B^{2}}\right] \\
& \left.+\gamma_{\nu} \sin 2 \beta \exp \left[-\frac{\left(\nu-\nu_{0}-\nu_{+}\right)^{2}}{B^{2}}\right] \cos (2 \pi \nu \tau+\delta)\right\}
\end{aligned}
$$


where $\nu_{+}=\left(\nu_{1}+\nu_{2}\right) / 2, \quad \nu_{-}=\left(\nu_{1}-\nu_{2}\right) / 2$, and $\gamma_{\nu}=$ $\exp \left(-\nu_{-}^{2} / B^{2}\right)$. After post-selection, the beams reflected from each FBG interfere [15]. As a result, there is a reshaping of the output spectrum.

Both FBG can show slightly different spectral responses due to errors in the fabrication process. We keep one of the FBGs at a constant temperature $T_{2}$, and measure its reflectivity spectrum to be centered at $\nu_{2}^{0}$. The other FBG is used to measure a variable temperature $T_{1}$, and the centroid of the spectrum of the reflected signal is assumed to change linearly with temperature as

$$
\nu_{1}\left(T_{1}\right)=\nu_{1}^{0}+\kappa\left(T_{1}-T_{2}\right) .
$$

The centroid of the spectrum at the output port of the interferometer is $\langle\nu\rangle=\int \nu S(\nu) \mathrm{d} \nu / \int S(\nu) \mathrm{d} \nu$, where $S$ is measured with the OSA. In the weak coupling regime, the temporal delay $\tau$ in the interferometer is much smaller than the pulse duration $T$, and the frequency shifts $\nu_{1}\left(T_{1}\right)$ and $\nu_{2}^{0}$ are small compared to the bandwidth $B$. In this scenario, the centroid of the spectrum writes

$$
\langle\nu\rangle=\nu_{0}+\nu_{+}+\mathcal{A} \nu_{-}-\mathcal{B},
$$

where

$$
\mathcal{A}=\frac{\cos 2 \beta}{1+\gamma_{\nu} \gamma_{\tau} \sin 2 \beta \cos \delta},
$$

is the amplification factor, $\gamma_{\tau}=\exp \left(-\pi \tau^{2} B^{2}\right)$, and the last term in Eq. (4) is given by

$$
\mathcal{B}=\frac{\pi \gamma_{\nu} \gamma_{\tau} \tau B^{2} \sin 2 \beta \sin \delta}{1+\gamma_{\nu} \gamma_{\tau} \sin 2 \beta \cos \delta} .
$$

We take as reference for the measurements $\nu_{2}^{0}$, which is measured for an angle $\beta=-90^{\circ}$. One can easily find from Eqs. (3) and (4) that the shift of the centroid $\langle\Delta \nu\rangle=\langle\nu\rangle-\nu_{0}-\nu_{2}^{0}$ is

$$
\langle\Delta \nu\rangle=\frac{\kappa}{2}(\mathcal{A}+1)\left(T_{1}-T_{2}\right)+(\mathcal{A}+1)\left(\frac{\nu_{1}^{0}-\nu_{2}^{0}}{2}\right) .
$$

The shift of the centroid of the spectrum is proportional to the difference in temperature between the FBGs. If we project into a polarization state that selects only the signal coming from the FBG with a variable temperature $\left(\beta=0^{\circ}\right)$, then $\mathcal{A}=1$, and the constant of proportionality turns out to be $\kappa$, which is determined by the response of the FBG to changing temperatures. However, when we project into different polarization states, $\kappa$ is multiplied by the amplification factor $\mathcal{A}$ that can be much larger than one. Figure 1(b) shows the shift of the centroid of the spectrum, expressed in terms of wavelength shift, for three values of the amplification factor that corresponds to three different output polarization projections. Notice the large enhancement of wavelength shift that can be achieved for a given temperature difference $T_{1}-T_{2}$ when different output polarization states are selected.

The amplification factor can be very large. As it can be seen in Eq. (5), the closer is the value of the factor $\gamma_{\nu} \gamma_{\tau} \cos \delta$ to 1 , the larger is the value of the amplification factor. Inspection of Fig. 1(c) shows that an amplification factor of up to nearly 80 can be achieved for $\gamma_{\nu} \gamma_{\tau} \cos \delta=0.9999$. Larger values of the amplification factor can indeed be obtained. However, in practice, its maximum value is limited by different experimental factors. On the one hand, it strongly depends on how well the phase introduced by the single-mode fibers $\phi$ is compensated by the variable retarder (LCVR), as shown in Fig. 1(c). From Eq. (5), we obtain that the maximum amplification factor that can be achieved for an uncompensated phase is $\mathcal{A}_{\max }=\left(1-\cos ^{2} \delta\right)^{-1 / 2}$, which is obtained for the post-selection angle $-\pi / 4^{\circ} \pm \beta_{\max }+\frac{1}{2} \arcsin (\cos \delta)$. The largest enhancement is obtained when $\phi=\Gamma$. On the other hand, in any weak value amplification scenario, there is attenuation of the amplitude of the output signal. For low amplification factors, this attenuation can be similarly small. However, a large amplification factor is accompanied by a large attenuation, since the input and post-selected polarization are nearly orthogonal. The amplification factor achievable is thus limited by the signal-to-noise ratio available at the detection stage.

Figure 2 depicts the spectra of the signals reflected from $\mathrm{FBG}_{1}\left(\beta=-90^{\circ}\right)$ and $\mathrm{FBG}_{2}\left(\beta=0^{\circ}\right)$. Figure $2(\mathrm{a})$ corresponds to the signal reflected from $\mathrm{FBG}_{2}$ at a fixed temperature, while Fig. 2(b) corresponds to the reflection from $\mathrm{FBG}_{1}$ at different temperatures. The spectrum of the signal reflected from each FBG is composed of a principal lobe $\sim 2.5 \mathrm{~nm}$ wide (FWHM), and a side lobe with smaller amplitude that appear as a result of the high contrast in index of refraction in the gratings. Since our scheme relies on the measurement of the centroid of a Gaussian-like spectra given a post-selection, the presence of non-negligible side lobes can alter the measurements. To avoid this effect, each measured spectrum is filtered numerically using a super-Gaussian filter indicated by the dashed line in Fig. 2.

To measure the spectrum, the OSA measured 1000 points in a range of $14 \mathrm{~nm}$ around $1551 \mathrm{~nm}$, measuring thus the spectrum with a 14-pm resolution. The estimation of the centroid of the signal was done in a time of $2 \mathrm{~s}$, which included acquisition of data, saving, and processing. This is in no way a fundamental limit for the measurement time. Faster detection, by reducing the bandwidth, and faster electronic processing can substantially reduce the overall time needed for a measurement.

Figure 3(a) presents the measured shift of the centroid of the output spectrum for $T_{1}-T_{2}=11^{\circ} \mathrm{C}$ and different post-selection angles (dots). For small angle deviations around $\beta=-45^{\circ}$, shifts of the centroid of the spectrum up to $\pm 0.6 \mathrm{~nm}$ are observed. This corresponds to a three-fold enhancement with respect to the initial shift of $0.19 \mathrm{~nm}$ given by the FBGs with no weak amplification scheme. The solid line indicates the best theoretical fit obtained using Eq. (7). Figure 3(b) shows some selected spectra measured after performing the super-Gaussian filtering. In general, there is a trade-off between the centroid shift observable for a specific temperature difference and the
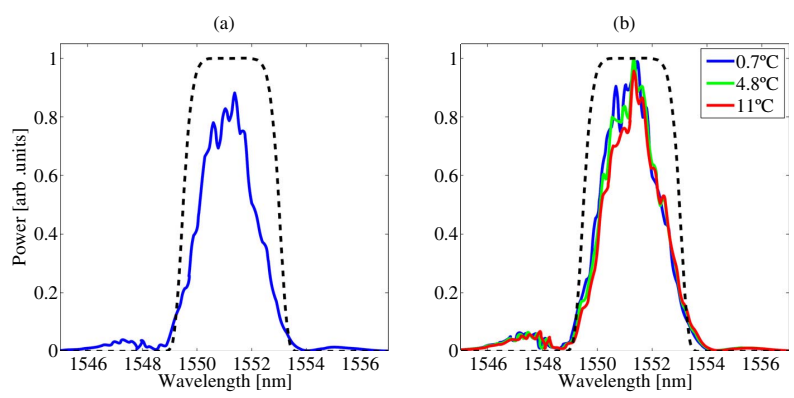

Fig. 2. (a) Spectrum of the signal reflected from $\mathrm{FBG}_{2}$ at a fixed temperature $\left(\beta=-90^{\circ}\right)$, and (b) from $\mathrm{FBG}_{1}$ at different temperatures $\left(\beta=0^{\circ}\right)$. Dashed lines: transmission function of the super-Gaussian filter used to get rid of the unwanted side lobes present in the signal. 

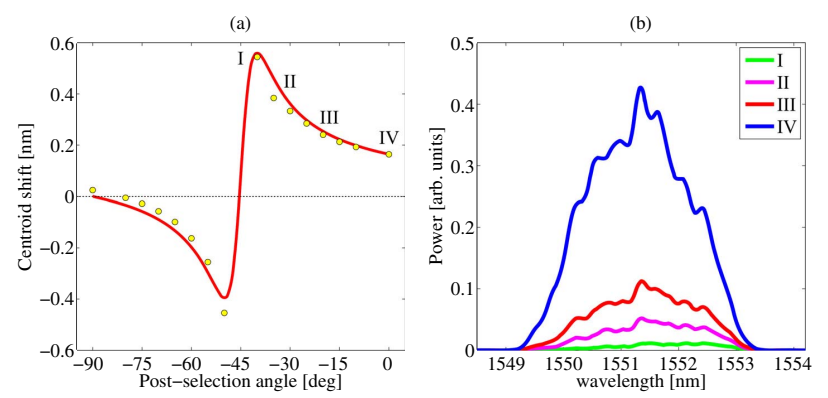

Fig. 3. (a) Shift of the centroid of the spectrum for $T_{1}-T_{2}=$ $11^{\circ} \mathrm{C}$ and different post-selection angles (dots) in the interval $-90^{\circ} \leq \beta \leq 0^{\circ}$. (b) Output spectrum measured for some selected cases: $\beta=-40^{\circ}$ (I), $\beta=-35^{\circ}$ (II), $\beta=-25^{\circ}$ (III), and $\beta=0^{\circ}$ (IV).

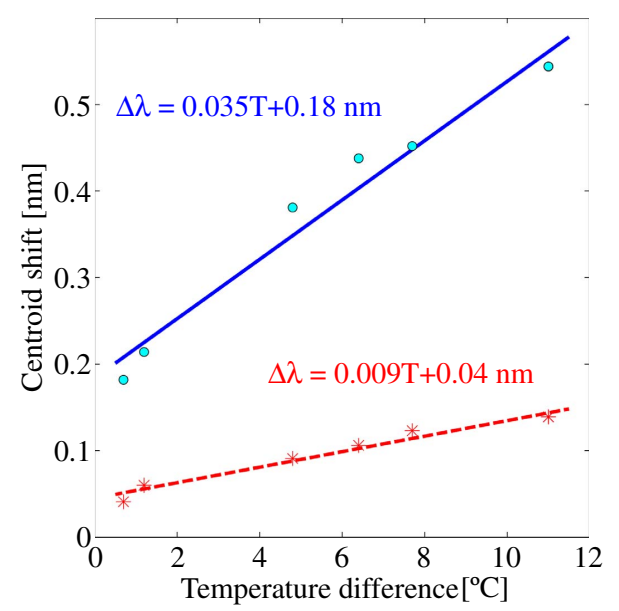

Fig. 4. Variation of the centroid of the output spectra as a function of the temperature difference for a fixed post-selection angle. The case $\beta=0^{\circ}$ (asterisks) illustrates the situation where no WVA scheme is used. The case $\beta=-40^{\circ}$ (circles) shows the case where the centroid shift is enhanced by a factor of $\approx 4$ by means of the WVA scheme.

amount of losses that can be tolerated to keep a good SNR. In our scheme, the side lobes become relevant with respect to the main lobe of the output spectrum for post-selection angles within the interval $-50^{\circ} \leq \beta \leq-40^{\circ}$. For this reason, a maximum amplification factor of $\approx 4$ is obtained for such angles.

Figure 4 shows the measured variation of the centroid position of the output spectra as a function of the temperature difference for a given post-selection. Circles indicate the case when the output signal is projected into a polarization state with $\beta=-40^{\circ}$, so that the output spectrum centroid drifts $\sim 0.035 \mathrm{~nm} /{ }^{\circ} \mathrm{C}$ (solid line). For the sake of comparison, asterisks show the case where no weak amplification is used $\left(\beta=0^{\circ}\right)$, generating a spectrum centroid variation of $\sim 0.009 \mathrm{~nm} /{ }^{\circ} \mathrm{C}$ (dashed line). The use of the WVA provides a four-fold enhancement of the sensitivity.

The existence of a temperature-dependent group delay between two orthogonal polarizations used to interrogate the FBGs is the key enabling element in WVA. Here we chose to generate the group delay by illuminating two FBGs embedded in a Mach-Zehnder interferometer. Environmental perturbations may affect the measurement, since any measurement based on interferometers requires a precise control. Therefore, in terms of applications, the present scheme is intended for short-range applications like health applications and metrology. However, for an out-of-the-lab implementation of the technique, we are exploring more simple schemes using tailored FBGs that will allow an all-fiber solution to generate two orthogonally polarized reflected signals with a measurable temperature-dependent delay.

In conclusion, we have demonstrated that WVA can be used to enhance the sensitivity of sensors based on fiber Bragg gratings. The shift of the spectrum of the signal reflected by a FBG due to temperature changes was measured to be $\sim 0.009 \mathrm{~nm} /{ }^{\circ} \mathrm{C}$. With a weak amplification scheme, we measured a change of $0.035 \mathrm{~nm} /{ }^{\circ} \mathrm{C}$, a fourfold increase. The proposed scheme is compatible with wavelength-division multiplexing (WDM) and time-division multiplexing (TDM) being possible to obtain a quasi-distributed measurement. Besides, WVA is compatible with the use of other schemes that enhance the sensitivity of FBGs sensors, such as the use of extrinsic FBG structures. In this way, WVA can multiply the resolution gained with the help of the extrinsic FBG structures. Therefore, in scenarios where the measurable shift of the spectrum is limited by the detection stage, but the decrease of signal energy that accompanies still keeps the signal-to-noise ratio at an usable level, weak value amplification is a promising scheme to enhance the capabilities of FBG-based sensor systems.

Funding. Severo Ochoa program; Fundació Privada Cellex, Barcelona; Research Excellency Award Program GVA PROMETEO 2013/012; Spanish MCINN (TEC201453727-C2-1-R).

Acknowledgement. D. B., S. S., and J. C. acknowledge financial support from the Spanish MCINN and the Research Excellency Award Program GVA PROMETEO 2013/012.

\section{REFERENCES}

1. R. Kashyap, Fiber Bragg Gratings (Academic, 1999).

2. Y. Aharonov, D. Z. Albert, and L. Vaidman, Phys. Rev. Lett. 60, 1351 (1988).

3. I. M. Duck, P. M. Stevenson, and E. C. G. Sudarhshan, Phys. Rev. D 40, 2112 (1989).

4. J. C. Howell, D. J. Starling, P. Ben Dixon, P. K. Vudyasetu, and A. N. Jordan, Phys. Rev. A 81, 033813 (2010).

5. J. P. Torres, G. Puentes, N. Hermosa, and L. J. Salazar-Serrano, Opt. Express 20, 18869 (2012).

6. N. W. Ritchie, J. G. Story, and R. G. Hulet, Phys. Rev. Lett. 66, 1107 (1991).

7. O. Hosten and P. Kwiat, Science 319, 787 (2008).

8. P. Ben Dixon, D. J. Starling, A. N. Jordan, and J. C. Howell, Phys. Rev. Lett. 102, 173601 (2009).

9. J. C. Howell, D. J. Starling, P. B. Dixon, K. P. Vudyasetu, and A. N. Jordan, Phys. Rev. A 82, 063822 (2010).

10. X.-Y. Xu, Y. Kedem, K. Sun, L. Vaidman, C.-F. Li, and G.-C. Guo, Phys. Rev. Lett. 111, 033604 (2013).

11. L. J. Salazar-Serrano, D. Janner, N. Brunner, V. Pruneri, and J. P. Torres, Phys. Rev. A 89, 012126 (2014).

12. B. A. Tahir, J. Ali, and R. A. Rahman, Int. J. Mod. Phys. B 23, 2349 (2009).

13. A. L. Ricchiuti, D. Barrera, K. Nonaka, and S. Sales, Opt. Lett. 39, 5729 (2014)

14. P. Egan and J. A. Stone, Opt. Lett. 37, 4991 (2012).

15. L. J. Salazar-Serrano, A. Valencia, and J. P. Torres, Opt. Lett. 39, 4478 (2014). 